\title{
A New Storage Scheme for Approximate Location Queries in Object-Tracking Sensor Networks
}

\author{
Jianliang Xu, Member, IEEE, Xueyan Tang, Member, IEEE, and Wang-Chien Lee, Member, IEEE
}

\begin{abstract}
Energy efficiency is one of the most critical issues in the design of wireless sensor networks. Observing that many sensor applications for object tracking can tolerate a certain degree of imprecision in the location data of tracked objects, this paper studies precision-constrained approximate queries that trade answer precision for energy efficiency. We develop an Energy-conserving Approximate StoragE (EASE) scheme to efficiently answer approximate location queries by keeping error-bounded imprecise location data at some designated storage node. The data impreciseness is captured by a system parameter called the approximation radius. We derive the optimal setting of the approximation radius for our storage scheme based on the mobility pattern and devise an adaptive algorithm to adjust the setting when the mobility pattern is not available a priori or is dynamically changing. Simulation experiments are conducted to validate our theoretical analysis of the optimal approximation setting. The simulation results show that the proposed EASE scheme reduces the network traffic from a conventional approach by up to 96 percent and, in most cases, prolongs the network lifetime by a factor of $2-5$.
\end{abstract}

Index Terms-Energy efficiency, data dissemination, data storage, location query, wireless sensor network.

\section{INTRODUCTION}

$\mathrm{O}$ WING to the rapid advances in sensing and wireless communication technologies, wireless sensor networks have emerged as a promising solution for a wide range of civil and military applications. In this paper, we consider object-tracking sensor networks, one of the most important classes of sensor networks. Example applications of object tracking include wildlife animal monitoring in remote areas and intrusion detection on military sites. Users in these applications are interested in location queries, which return the locations of tracked moving objects.

A sensor network is typically composed of a large number of tiny sensor nodes equipped with data processing, sensing, and communication capabilities. The sensor nodes usually operate in an unattended manner and are battery powered. However, replacing the battery is not only costly but also impossible in many situations (for example, in a hard-to-reach area). Thus, energy efficiency is a critical consideration in the design of large-scale sensor networks. There has been significant research on energy-conserving object-tracking sensor networks (for example, [9], [10], [35]). Most of these studies aimed at reducing the number of

- J. Xu is with the Department of Computer Science, Hong Kong Baptist University, Kowloon Tong, KLN, Hong Kong.

E-mail:xujl@comp.hkbu.edu.hk.

- X. Tang is with the School of Computer Engineering, Nanyang Technological University, Nanyang Avenue, Singapore 639798.

E-mail: asxytang@ntu.edu.sg.

- W.-C. Lee is with the Department of Computer Science and Engineering, The Pennsylvania State University, University Park, PA 16802.

E-mail:wlee@cse.psu.edu.

Manuscript received 5 Dec. 2006; revised 26 Mar. 2007; accepted 13 June 2007; published online 6 July 2007.

Recommended for acceptance by I.-P. Sheu.

For information on obtaining reprints of this article, please send e-mail to tpds@computer.org, and reference IEEECS Log Number TPDS-0393-1206.

Digital Object Identifier no. 10.1109/TPDS.2007.70740. sensor nodes activated for tracking an object and/or reducing the location update traffic in providing accurate answers to location queries. ${ }^{1}$

Imprecision is an inherent property of object-tracking sensor networks. State-of-the-art location positioning technologies such as GPS and triangulation are not error-free. Moreover, many applications are willing to tolerate a certain degree of imprecision or error in the data due to either the application nature or the high resource constraints in sensor networks. As such, here, we take a different approach to improve energy efficiency by exploiting the trade-off between data quality and energy conservation. Instead of always feeding the most accurate answers to location queries, we investigate the problem of providing precision-constrained approximate locations based on user tolerances. In our model, an approximate location query is specified by an object identifier and a precision constraint. The sensor network responds with a location bounded by the required precision.

Inspired by [11] and [23], we develop an Energyconserving Approximate StoragE (EASE) scheme to efficiently answer approximate location queries. Whereas most prior work assumed centralized/designated storage for data collection and query answering [9], [17], [32], EASE innovatively maintains two versions of object location data in the network. High-precision data are kept at some local storage node close to a moving object in order to reduce longdistance traffic resulting from remote updates. Meanwhile, the same data with a lower precision are replicated at some designated storage node that is known to users in order to reduce the query traffic. In the EASE scheme, the imprecision of location data at the designated storage node is

1. The accuracy is achieved by best effort since the object location cannot be 100 percent accurate due to network delay, discrete sampling instances, etc.

Published by the IEEE Computer Society 
bounded by an approximation radius, which specifies a geographical area in which the low-precision location data are considered valid. In other words, a location update due to object movement will not be sent to the designated storage node if the object remains within the approximation radius. Correspondingly, a query is answered by the designated storage node if its precision constraint is weaker than what is specified by the approximation radius. Otherwise, the query is forwarded to the local storage node for resolution. As such, the EASE scheme attempts to optimize the network performance (in terms of reducing network traffic and energy consumption) by balancing the update traffic and query traffic. This is achieved by properly setting the approximation radius. We derive the optimal setting of the approximation radius based on the mobility pattern and also devise an adaptive algorithm to adjust the setting on the fly when the mobility pattern is unknown or is dynamically changing. We show via simulation experiments that the EASE scheme, working together with the proposed methods for setting the approximation radius, reduces the network traffic by up to 96 percent from a conventional approach and, in most cases, prolongs the network lifetime by a factor of 2-5.

We summarize the contributions made in this study as follows:

- To the best of our knowledge, this is the first study on data dissemination in object-tracking sensor networks that attempts to address the issue of energy efficiency by exploiting the trade-off between data quality and energy conservation.

- An energy-efficient storage scheme, called EASE, is proposed to efficiently answer precision-constrained approximate location queries.

- The setting of the proposed storage scheme is analyzed and optimized through a theoretical study. The theoretical analysis is validated by simulation experiments.

- An adaptive algorithm is proposed to adjust the setting of the approximation radius for unknown/ dynamic workloads.

- An extensive performance evaluation is conducted to evaluate the performance of the proposed EASE scheme and the methods for setting the approximation radius.

The rest of this paper is organized as follows: Section 2 reviews related work. The system model is described in Section 3. Section 4 presents the proposed EASE scheme in detail. We analyze the performance of EASE and investigate the setting of the approximation radius in Section 5 . Section 6 presents the results of the performance evaluation. Finally, Section 7 concludes the paper.

\section{Related Work}

\subsection{Object-Tracking Sensor Networks}

There are two research directions for improving the lifetime of an object-tracking sensor network. One is to reduce energy consumption in the sensing component (for example, [18] and [35]). The basic idea is to activate only the essential sensor nodes needed to track the moving objects while leaving the other nodes in a power-saving mode. In [31] and [33], the sensor nodes are organized into a cluster-based architecture such that a cluster head calculates object locations based on signal readings from its slave nodes. Based on these studies, we assume that object locations can be obtained by cluster heads and only focus our task on where and how to store the location data in support of energy-efficient approximate location queries.

The other direction, aligned with ours, is to improve energy efficiency by reducing network traffic in disseminating location updates. Nevertheless, the focuses of the prior studies are different from ours. Goel and Imielinski [9] proposed a prediction-based approach. A base station collects sensor readings and periodically generates predictions to be sent back to the sensor nodes. A sensor node reports a location update only when its reading differs from the predicted one. $\mathrm{Xu}$ et al. [32] suggested a dual-prediction scheme where a fixed prediction model is deployed at both the base station and the sensor nodes. These studies complement our work in that prediction can be incorporated into our EASE scheme to further reduce remote update traffic. Kung and Vlah [17] investigated continuous location queries and proposed a publish-and-subscribe tracking method. In contrast, we leverage error tolerances to improve network performance for one-shot location queries. Although there has been research on the trade-off between energy conservation and tracking quality (for example, [10], [24]), the trade-off has not been investigated in the dissemination of location data, which is the topic of this paper.

Our work also bears some similarity to location management for mobile networks [4]. As the purpose of location management is to locate roaming users for call delivery, locations are managed at a fixed granularity (that is, cell). In contrast, our architecture is capable of adaptively storing location data at different degrees of accuracy to improve the efficiency of query processing.

\subsection{Data Storage and Query Processing}

A simple storage model is to have a centralized base station collect and store the sensed data. This approach is good for aggregate data collection (for example, sum, average, maximum, and median) [21], [28], where excessive sensed data can be pruned during aggregation along the routing path, and only short summaries are maintained at the base station. However, this is not efficient for nonaggregate data collection (for example, the location queries considered in this paper). The base station and the sensor nodes around it can easily become hotspots, which would shorten the network lifetime.

Recently, in-network storage has been advocated in many research projects. In the TinyDB project, Madden et al. [20] presented the pull-based acquisitional query processing (ACQP), where the sensor nodes control where, when, and how often data are acquired and delivered to query operators. The Cougar project [7] employed a hybrid pull-push model, in which sensed data are pushed to some selected view nodes, from where the data are pulled to answer queries. Ratnasamy et al. [26] proposed an in-network data-centric storage (DCS) model: sensed data are pushed to the sensor node nearest to some geographical location hashed from a predefined key. Zhang et al. [36] suggested storing sensed data locally. A centric ring-based index was proposed to facilitate query processing. More recently, Lu et al. [19] proposed a spatiotemporal query service called MobiQuery to allow 
mobile users to query their surrounding areas through a sensor network. Jiang and Jin [14] developed robust aggregation techniques for extracting statistical information from sensor networks. Unfortunately, none of these prior studies have examined the ability of approximate data storage to improve energy efficiency.

\subsection{Approximate Query Processing}

Early work on approximate query processing focused on a wired network [23]. Han et al. [11] conducted a pioneering study on answering approximate queries in sensor networks. They developed an efficient data collection protocol to fulfill the application-specified data quality while minimizing the energy consumption of sensor nodes. However, the solutions developed in [11] are not applicable to object-tracking applications. This is because they considered only a simple single-hop system where each sensor node communicates with the server directly, and each target phenomenon is always captured by a fixed sensor. In contrast, we consider a dynamic sensing scenario where the location of a moving object is acquired by different nodes at different times; thus, a cooperative location updating protocol is needed. Moreover, we consider a multihop sensor network. As a result, the cost of location updates/queries also varies according to the locations of moving objects. These differences make our system modeling and performance analysis completely different from those in [11].

Precision-constrained queries [6], [27], [30] have also been studied for in-network data aggregation, which has a different focus from object-tracking sensor networks. Compressing historical sensor readings for transmission also saves energy [5]. However, it is applicable to querying historical data only. In contrast, we consider applications that are interested in querying the current locations of moving objects.

\subsection{Data Routing}

A sensor network is typically connected by wireless links in an ad hoc manner. To relay data in a sensor network, many routing algorithms have been proposed to address energy efficiency, scalability, and reliability issues. They can be classified into three categories: data-centric, hierarchical, and location-based routings [3]. In data-centric routing (for example, directed diffusion [13]), the sink floods the query to a certain region of interest, and the sources report data to the sink through the route established based on the named query. Due to the high cost of flooding, such a protocol is suitable for long-lived queries only. Hierarchical protocols (for example, LEACH [12]) group the sensor nodes into clusters such that a cluster head performs data aggregation/fusion and communicates with other heads on behalf of the nodes within its cluster. Location-based protocols (for example, greedy perimeter stateless routing (GPSR) [15]) make use of geographical position information to transport data.

\section{System Model}

We consider a sensor network consisting of a large number of stationary sensor nodes deployed in some operational area. Each sensor node is aware of its own location, through GPS for example. We assume that the nodes organize themselves into clusters and that every cluster has a cluster head. A cluster head is more powerful than an ordinary sensor node. It is equipped with some local storage to store data and is also capable of communicating with other cluster heads to exchange data. The sensor nodes in a cluster can work together to recognize and track the objects in their vicinity; for example, a cluster head can triangulate object locations based on signal readings from its slave nodes [31], [29], [33]. The object locations are sampled at a fixed sampling rate. We also assume that each moving object being tracked has a unique identifier. Since this paper aims at energy-efficiently storing and disseminating object location data in support of approximate location queries, we shall focus on reducing communication among cluster heads. Unless explicitly specified, a sensor node refers to a cluster head in the rest of this paper.

Approximate location queries. The sensor network under consideration supports a large number of users making one-shot queries for the locations of moving objects. The queries can be made via a sensor node (known as the querying node) from anywhere in the network. Each approximate location query is specified by a tuple $<$ object_id, $p\rangle$, where object_id is the identifier of the target object, and $p$ is the error in object location that the query can tolerate. $^{2}$

Local storage (LS) and centralized storage (CS). Intuitively, the object location data can be stored at 1) the sensor node that detected the object or 2) a centric storage node, which could be either a centralized base station or a sensor node determined by the DCS scheme [26] (see Fig. 1a). ${ }^{3}$ If the location data are stored locally at the detecting node (known as LS), a query that wants to find the location of some object has to be flooded over the whole network. Thus, the query cost is high. In contrast, if the CS scheme is adopted in the system, any location update of a moving object should be sent to the centric storage node. This results in high update traffic. In the next section, we propose a hybrid in-network storage scheme that achieves a good balance between the query cost and the update cost for approximate location queries. Without loss of generality, we shall assume for the rest of this paper that the DCS scheme [26] is employed for CS.

\section{EASE: Energy-Conserving Approximate STORAGE}

This section proposes the new EASE scheme that takes advantage of the error tolerances of queries. We first give an overview of EASE in Section 4.1. Section 4.2 describes the location updating protocol for working with EASE. Finally, we discuss how EASE handles node failures and message losses in Section 4.3.

\subsection{Overview}

The EASE scheme attempts to cut down the update traffic by maintaining two versions of the location data for each object: an accurate version at a local storage node and an

2. For simplicity, we assume in this paper that $p$ is the error tolerance in addition to the unavoidable system error such as the inaccuracy of the positioning technique. In practice, $p$ can be determined by the user's error tolerance minus the maximum system error.

3. In DCS, the centric storage node of an object is determined by applying a predefined hash function to the object identifier. 


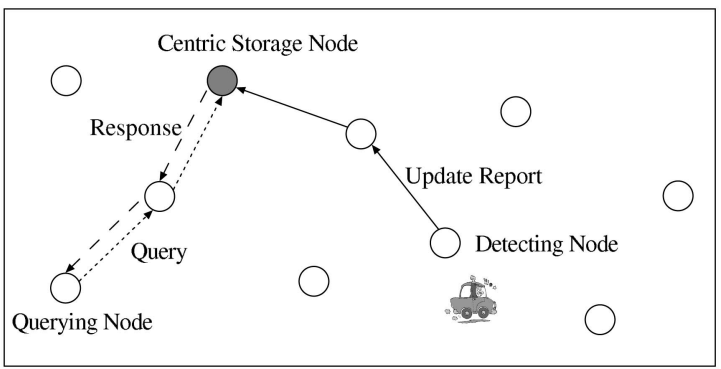

(a)

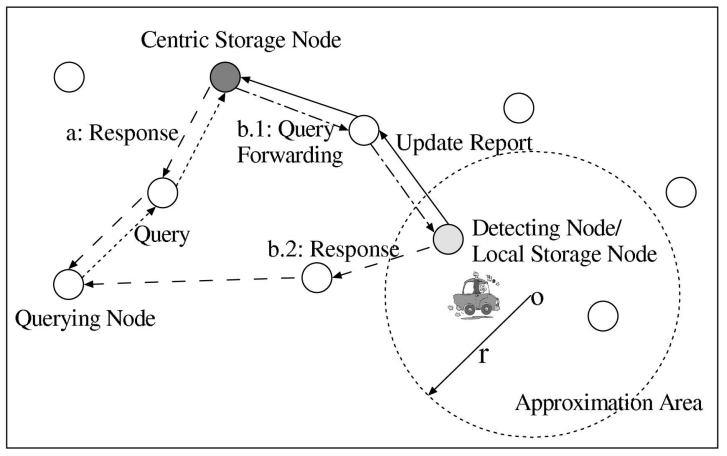

(b)

Fig. 1. Illustration of a query processing procedure. (a) CS. (b) EASE.

approximate version at the centric storage node. The approximate location of an object at the centric storage node is bounded by an approximation radius. A stored location $o$ with an approximation radius $r$ means that the object must be in an approximation area defined by a circle of radius $r$ centering at $o$.

In addition to storing the approximation location of an object, the centric storage node also keeps track of the local storage node of the object. That is, the centric storage node maintains two attributes for an object: the approximate location and the local storage node. The former is used to answer queries with less stringent precision constraints, whereas the latter is used to forward queries with more stringent precision constraints (without this information, the local storage nodes can only be found by flooding). Upon receiving an approximate location query with an error tolerance $p$, the centric storage node compares it against the approximation radius $r$ of the stored location. If $p \geq r$, the stored location satisfies the precision requirement and, hence, it is returned to the querying node immediately (as shown by case $a$ in Fig. 1b). If $p<r$, the stored location is inadequate in precision. Consequently, the query is forwarded to the local storage node, and the result is returned from that node (as shown by case $b$ in Fig. 1b).

\subsection{Location Updating Protocol}

Initially, the local storage node of an object is the node that first detected it. Every sensor node in the approximation area is notified of the local storage node. At each subsequent sampling instance, if the object location detected is within its current approximation area, the detecting node sends a local update including the new object location to the local storage node, as shown in Fig. 2a. Otherwise, if the object moves out of the current approximation area, the detecting node elects itself as the new local storage node and sends a remote update including its own

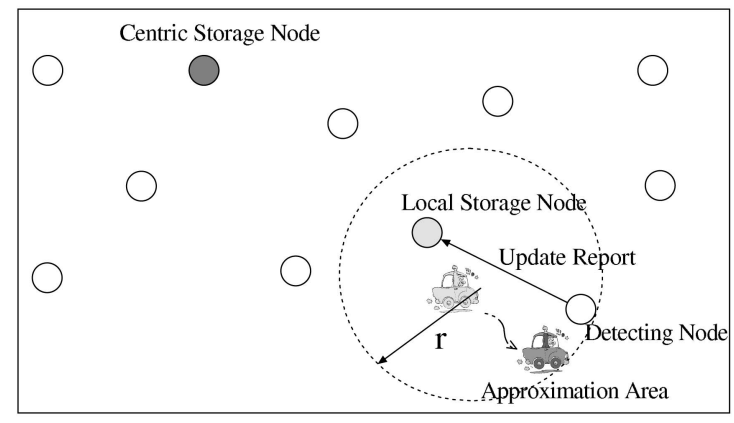

(a)

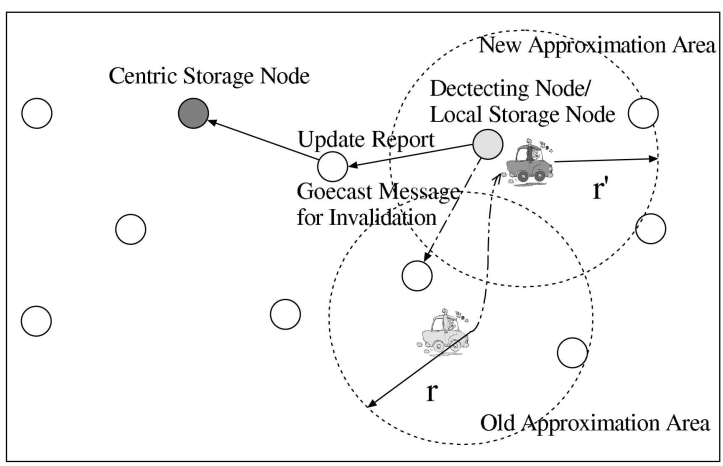

(b)

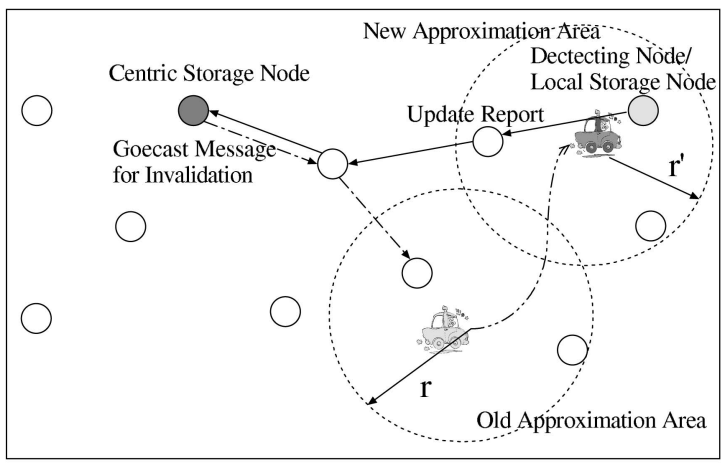

(c)

Fig. 2. Location update dissemination with EASE. (a) Local update. (b) Remote update: case 1. (c) Remote update: case 2.

ID/location and the new object location to the centric storage node (see Figs. 2b and 2c). It is likely that the object is still in the current approximation area, but the detecting node is not aware of the current local storage node (for example, the detecting node is outside the approximation area). In this case, the detecting node also elects itself as the new local storage node and sends a remote update to the centric storage node. A new approximation area is then formed as a circle centered at the new object location.

To notify the sensor nodes in the new approximation area, the new local storage node sends out a notification geocast [22] message including its own ID/location and the new approximation area (see below for how it works). If the new local storage node is aware of the previous local storage node (and, hence, the obsolete approximation area), it also sends an invalidation geocast message to the sensor nodes in the obsolete approximation area to invalidate the recorded local storage node (see Fig. 2b). Otherwise, if the new local storage node is not aware of the previous local 
storage node, it informs the centric storage node about this in the remote update and asks the centric node to invalidate the obsolete approximation area using geocast (see Fig. 2c). Given an approximation radius $r$, Algorithms 1 through 3 summarize the protocols executed at the centric storage node, the local storage node, and the detecting node, respectively.

Algorithm 1. Protocol executed at the centric storage node.

1: if receiving a query $<$ object_id, $p>$ then

2: $\quad$ if $p \geq r$ then

3: $\quad$ return the stored location to the querying node;

4: $\quad$ else

5: forward the query to the local storage node of the queried

object;

6: end if

7: end if

8: if receiving a remote location update message then

9: if the old approximation area is unknown at the local storage node then

10: $\quad$ send an invalidation geocast message to the old approximation area;

11: end if

12: store the new location and local storage node of the object;

13: end if

Algorithm 2. Protocol executed at the local storage node.

1: if receiving a forwarded query $\langle$ object_id, $p\rangle$ then

2: return the stored location to the querying node;

3: end if

4: if receiving a local location update message then

5: store the new location of the object;

6: end if

Algorithm 3. Protocol executed at a node detecting an object located at $o$.

1: if $o$ is in the valid approximation area and the local storage node is known then

2: $\quad$ send a location update to the local storage node;

3: else

4: $\quad$ send a location update to the centric storage node; piggyback the information whether the old approximation area is known or not;

5: elect itself as a new local storage node;

6: $\quad$ set the new approximation area $A^{\prime} \leftarrow$ a circle centered at $o$ with radius $r$;

7: $\quad$ if the old approximation area $A$ is known then

8: $\quad$ send an invalidation geocast message to $A$;

9: $\quad$ end if

10: $\quad$ send a notification geocast message to $A^{\prime}$;

11: end if

The purpose of geocast is to send a message to all the nodes in a given geographical area. It works in two phases. In the first phase, the message is routed toward the target area using some geographical routing protocol such as GPSR [15]. If the geocast initiator is within the target area, the first phase is not needed. After reaching the target area, the message is flooded to all sensor nodes in the area through broadcast. On receiving the message, a sensor node further broadcasts it to the neighbors only if the message has not been received before and the sensor node is within the target area. Recall that in EASE, when the local storage node changes, a notification message and an invalidation message are geocast to the new and obsolete approximation areas, respectively. In order to avoid redundant message broadcast in the overlap region of these two areas, we do not distinguish the notification message from the invalidation message in implementation. A geocast message specifying both the obsolete and new approximation areas are sent to these two areas at the same time. On receiving the geocast message, if the node is in the new approximation area, it records the new local storage node. Otherwise, if the node is in the obsolete approximation area, it removes the recorded old local storage node.

\subsection{Discussion}

Wireless sensor networks are unreliable in nature. In this section, we discuss the strategies that EASE can employ to deal with node failures and message losses. Note that these issues are faced by any data dissemination schemes including LS and CS.

The EASE scheme relies on a centralized base station or DCS to store approximate location data. To tolerate node failures, DCS enhances the centric storage nodes by replication [8], [26]. EASE stores accurate location data at local storage nodes, which can also be replicated to improve reliability and availability using similar techniques employed by DCS.

As the link loss rate is high in sensor networks, hop-by-hop recovery (for example, by link-level retransmission) has been suggested to remedy message losses [16]. In addition, message losses can be handled at the application layer. For example, for query messages, we set a TTL value (that is, twice the round-trip time between the two farthest nodes in the network). If a query is not answered within the TTL time, the application may assume that the previous query has got lost in the network and resend the query.

There are two types of geocast messages in EASE (that is, invalidation and notification). If a notification message is lost, the sensor network still functions properly. In the worst case, if a sensor node is not notified of the local storage node, upon detecting the object, it will elect itself as a new local storage node and send a remote update to the centric storage node. If an invalidation message is lost, some sensor nodes may not be aware of the change of local storage node. We can request the previous local storage node to acknowledge the receipt of the invalidation message. The new local storage node will retransmit the message until it is acknowledged. For other nodes in the obsolete approximation area, if they do not receive the invalidation message and continue to send the local update to the previous local storage node, the previous local storage node will notify them of the new local storage node.

\section{Performance Analysis and Optimization}

In this section, we first analyze the performance of EASE in terms of message complexity (Section 5.1). Then, we study the setting of the approximate radius for EASE. When the mobility pattern is available, we analyze the optimal setting of the approximation radius (Section 5.2). When the 
TABLE 1

Notations Used in Analysis

\begin{tabular}{ll}
\hline Notation & Description \\
\hline$n$ & number of sensor nodes \\
$\lambda$ & query rate \\
$\mu$ & sensor sampling rate \\
$C_{q n}$ & cost of answering a query by centric storage \\
$C_{q f}$ & cost of answering a query by local storage \\
$C_{u r}$ & cost of a remote update \\
$C_{u l}$ & cost of a local update \\
$r$ & approximation radius, i.e., the error bound \\
$f$ & sensor node density \\
$p_{\max }$ & maximum level of imprecision \\
$C(r)$ & overall complexity with approximation radius of $r$ \\
$p_{q f}(r)$ & probability of not being satisfied by centric storage \\
$\eta(r)$ & remote update rate \\
$d$ & moving distance of each step in random walk \\
$l$ & time taken to move a single step in random walk \\
\hline
\end{tabular}

mobility pattern is not available, we devise an adaptive algorithm to dynamically adjust the approximation radius (Section 5.3).

\subsection{Performance Analysis}

This section analyzes the performance of EASE in terms of message complexity (that is, the total number of message transfers in the network). A summary of the notations used in the analysis is provided in Table 1 . For simplicity, data transmission is assumed to be error-free. Assume that the query rate is $\lambda$ and the sensor sampling rate is $\mu$. Let $C_{q n}$ and $C_{q f}$ be the costs of answering a query by the centric storage node and the local storage node, respectively, and $C_{u r}$ and $C_{u l}$ be the costs of a remote update and a local update, respectively. Given an approximation radius of $r$, it is easy to see that the overall message complexity is given by

$$
\begin{aligned}
C(r)= & \left(1-p_{q f}(r)\right) \cdot \lambda \cdot C_{q n}+p_{q f}(r) \cdot \lambda \cdot C_{q f} \\
& +\eta(r) \cdot C_{u r}+\mu \cdot C_{u l},
\end{aligned}
$$

where $p_{q f}(r)$ is the probability that the approximate location stored at the centric storage node does not satisfy the queryspecified precision requirement, and $\eta(r)$ is the rate of remote updates (that is, the rate the object moves out of the approximation area). The four terms in the above formula represent the cost incurred by the queries answered by the centric storage node, the cost incurred by the queries answered by the local storage node, the cost of remote updates, and the cost of local updates, respectively.

Assume that the sensor network consists of $n$ uniformly distributed sensor nodes. As in [26] and [36], we use $n$ to approximate the cost of flooding a message over the whole network and $\sqrt{n}$ to approximate the cost of sending a message between two nodes in the network. It is easy to see (in Fig. 1b) that we obtain

$$
\begin{aligned}
& C_{q n}=2 \sqrt{n}, \\
& C_{q f}=3 \sqrt{n} .
\end{aligned}
$$

Note that the costs of $C_{u r}$ and $C_{u l}$ are a function of approximation radius $r$. We now derive these two costs and $p_{q f}(r)$. The rate of remote updates $\eta(r)$ depends on the mobility pattern and will be discussed in the next section.
A remote update involves the local storage node sending the update to the centric storage node and two geocast messages to notify (invalidate) the new (obsolete) approximation area. The remote update cost is approximated by $\sqrt{n}$. We approximate the geocast cost by the number of sensor nodes in the target area, that is, $\pi r^{2} f$, where $r$ is the approximation radius, and $f$ is the density of sensor nodes. Therefore, we obtain $C_{u r}$ as

$$
C_{u r}=\sqrt{n}+2 \pi r^{2} f .
$$

For a local location update, the average travel distance is given by

$$
\frac{\int_{0}^{r}(x \times 2 \pi x) d x}{\int_{0}^{r}(2 \pi x) d x}=\frac{2 / 3 \pi r^{3}}{\pi r^{2}}=\frac{2}{3} r .
$$

Thus, the local update cost can be approximated by the average number of sensor nodes encountered when traveling a distance of $\frac{2}{3} r$; that is

$$
C_{u l}=\frac{2}{3} r \sqrt{f} \text {. }
$$

We assume that the error tolerances of the queries are uniformly distributed in the range of $\left[0, p_{\max }\right]$. Thus, the probability of a query not being satisfied by the centric storage node is

$$
p_{q f}(r)=\min \left\{\frac{r}{p_{\max }}, 1\right\} .
$$

Combining (1) through (7), we can rewrite (1) as

$$
\begin{aligned}
C(r)= & \lambda \cdot 2 \sqrt{n}+\min \left\{\frac{r}{p_{\max }}, 1\right\} \cdot \lambda \cdot \sqrt{n} \\
& +\eta(r) \cdot\left(\sqrt{n}+2 \pi r^{2} f\right)+\mu \cdot \frac{2}{3} r \sqrt{f} .
\end{aligned}
$$

As can be seen, the overall message complexity is basically a function of approximation radius $r$. The next two sections study the setting of $r$ in detail.

\subsection{Optimal Approximation Setting with Known Mobility Pattern}

This section studies the optimal approximation setting based on the mobility pattern. We assume a two-dimensional random walk model [17], in which the object moves in steps. ${ }^{4}$ At each step, the object moves a distance of $d$ along an arbitrary direction (that is, with angle $\theta$ uniformly distributed in $[0,2 \pi])$. Each step takes a duration of $l$.

Let $T(r)$ be the average time an object takes to move out of a circle of radius $r$. With a random walk model, we have the following approximation (see the Appendix for details):

$$
T(r)=\left(\frac{r}{d}\right)^{2} \cdot l
$$

Therefore, the rate at which the object moves out of the circle (that is, the approximation area) is given by

$$
\eta(r)=\frac{d^{2}}{l r^{2}}
$$

4. The two-dimensional random walk model is used in this paper as a case study. The optimization technique presented here is applicable to other mobility patterns as long as their $\eta(r)$ can be estimated. 
Plugging (10) into (8), we get the following overall message complexity:

$$
\begin{aligned}
C(r)= & \lambda \cdot 2 \sqrt{n}+\min \left\{\frac{r}{p_{\max }}, 1\right\} \cdot \lambda \cdot \sqrt{n} \\
& +\frac{d^{2} \sqrt{n}}{l r^{2}}+\frac{2 \pi f d^{2}}{l}+\frac{2}{3} \mu r \sqrt{f} .
\end{aligned}
$$

Let $\frac{\partial C(r)}{\partial r}=0$. We obtain the optimal settings of $r^{*}$ in two cases:

$$
r^{*}= \begin{cases}\sqrt[3]{\frac{6 p_{\max } d^{2} \sqrt{n}}{l \cdot\left(3 \lambda \sqrt{n}+2 \mu p_{\max } \sqrt{f}\right)}} & \text { if } r \leq p_{\max }, \\ \sqrt[3]{\frac{3 d^{2} \sqrt{n}}{l \mu \sqrt{f}}} & \text { if } r>p_{\max }\end{cases}
$$

The one producing the lower message complexity $C(r)$ will be selected as the final setting of $r^{*}$. From (12), we can observe that the optimal setting of $r^{*}$ is affected by many factors such as the network size, query rate, sensor sampling rate, mobility pattern, and precision requirement. Intuitively, a faster movement (that is, larger $d$ or smaller $l$ ) results in a larger $r^{*}$ so as to reduce the remote update traffic; on the other hand, a smaller $r^{*}$ is desired at a higher sensor sampling rate (that is, higher $\mu$ ) in order to reduce the local update traffic. Also, note that if $r^{*}$ is set greater than $p_{\text {max }}$, all queries will be relayed to the local storage node via the centric storage node. In this case, the approximate data storage is used to maintain the location of the local storage node only.

\subsection{Adaptive Approximation Setting with an Unknown Mobility Pattern}

It is obvious that the larger the approximation radius $r$, the lower the rate of moving out of the approximation area $\eta(r)$. Following [11] and [23], we assume that $\eta(r)$ is proportional to $1 / r^{2}$; that is, $\eta(r)=\frac{K}{r^{2}}$, where $K$ is a parameter depending on the mobility model. We assume that $p_{\max }$ is sufficiently large, in which case $p_{q f}(r)=\frac{r}{p_{\max }}$. Using the optimization technique described in Section 5.2, we can derive the optimal $r^{*}$ for $r \leq p_{\max }$ :

$$
r^{*}=\sqrt[3]{\frac{6 p_{\max } K \sqrt{n}}{3 \lambda \sqrt{n}+2 \mu p_{\max } \sqrt{f}}} .
$$

It is not difficult to observe that when $r=r^{*}$,

$$
\frac{p_{q f}(r)}{\eta(r)}=\frac{6 \sqrt{n}}{3 \lambda \sqrt{n}+2 \mu p_{\max } \sqrt{f}}
$$

is a constant (denoted by $\rho$ ). Motivated by this observation, we propose an adaptive algorithm to dynamically adapt approximation radius $r$. The basic idea is to maintain the observed ratio of $p_{q f}(r)$ to $\eta(r)$ at $\rho$. To do so, the centric storage node keeps track of the query forwarding probability $p_{q f}(r)$. It also maintains the remote update rate using an exponential aging method. At each remote update, the estimate of the remote update rate is adjusted as

$$
\eta(r)^{\text {new }}=(1-\alpha) \cdot \eta(r)^{\text {old }}+\alpha \cdot \frac{1}{T_{c}-T_{l}},
$$

where $T_{c}$ is the current time, $T_{l}$ is the time of the last remote update, and $\alpha$ is a factor weighing the importance of the
TABLE 2

System Parameters and Settings

\begin{tabular}{l|l}
\hline Parameter & Setting \\
\hline \hline Field Size & $500 \times 500 \mathrm{~m}^{2}$ \\
Number of Nodes $(n)$ & 225 \\
Node Density $(f)$ & 1 node $/ 34 \times 34 \mathrm{~m}^{2}$ \\
Radio Range & $40 \mathrm{~m}$ \\
Power Consumption in Sending Messages & $60.0 \mathrm{~mW}$ \\
Power Consumption in Receiving Messages & $45.0 \mathrm{~mW}$ \\
Power Consumption in Idle Listening & $15.5 \mathrm{~mW}$ \\
Power Consumption in Sleeping & $0.09 \mathrm{~mW}$ \\
B-MAC Preamble Length & 271 bytes \\
B-MAC Wake-up Interval & $100 \mathrm{msec}$ \\
B-MAC Sampling Time & $2.55 \mathrm{msec}$ \\
Data Transfer Rate & $0.416 \mathrm{msec} / \mathrm{byte}$ \\
GPSR Beacon Interval & $3 \mathrm{sec}$ \\
GPSR Beacon Expiration & $13.5 \mathrm{sec}$ \\
GPSR Implicit Beacon & yes \\
Sensor Sampling Rate $(\mu)$ & $5 / \mathrm{sec}$ \\
Query Rate $(\lambda)$ & $0.1-10 / \mathrm{sec}$ \\
Maximum Level of Error Tolerance $\left(p_{\text {max }}\right)$ & $10-100 \mathrm{~m}$ \\
Query Message Payload Size & $10 \mathrm{bytes}$ \\
Update Message Payload Size & $40 \mathrm{bytes}$ \\
Result Message Payload Size & 40 bytes \\
Geocast Message Payload Size & $10 \mathrm{bytes}$ \\
Query Start Time & $20 \mathrm{sec}$ \\
Simulation Time & $500 \mathrm{sec}$ \\
\hline & \\
\hline &
\end{tabular}

current update against past updates. The setting of $r$ is then adjusted by the centric storage node based on Algorithm 4. Recall that $p_{q f}(r)$ is proportional to $r$, and $\eta(r)$ is inversely proportional to $r^{2}$. If the observed ratio is greater than $\rho \cdot(1+\epsilon)$, we decrease $r$ by a factor of $(1+\delta)$; if the observed ratio is lower than $\rho \cdot(1-\epsilon)$, we increase $r$ by a factor of $(1+\delta)$. On computing the new approximation radius, the centric storage node informs the new local storage node of the radius. On receiving the radius, the new local storage node performs the notification geocast (see Section 4.2). We shall evaluate the performance of this adaptive setting algorithm in the next section.

Algorithm 4. Adjustment of approximation radius (at the centric storage node).

1: for each remote update do

2: $\quad$ update the rate of remote updates $\eta(r)$ according to (15);

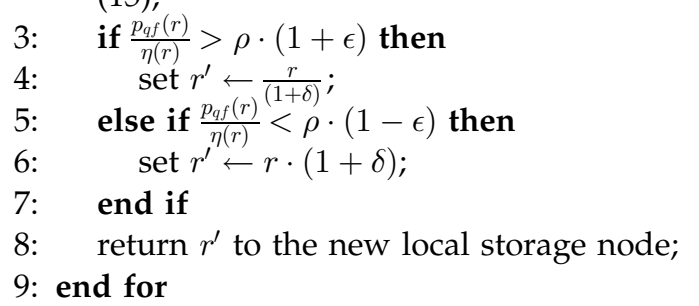

\section{Performance Evaluation}

\subsection{Simulation Setup}

In this section, we conduct simulation experiments to compare the proposed EASE scheme with the conventional storage schemes. We have developed a simulator based on ns-2 [1] and NRL's sensor network extension [2]. Table 2 summarizes the system parameters and their settings used in our experiments.

We simulate 225 sensor nodes as deployed in a $500 \times$ $500 \mathrm{~m}^{2}$ field. The field is divided into $22534 \times 34 \mathrm{~m}^{2}$ grid 
TABLE 3

Mobility Models and Parameter Settings

\begin{tabular}{l|c|c|c|c|c}
\hline $\begin{array}{l}\text { Mobility } \\
\text { Profile }\end{array}$ & \multicolumn{2}{|c|}{ Random / Semi-Random Walk } & \multicolumn{2}{c}{ Random Waypoint } \\
\cline { 2 - 6 } & $d$ & Init Speed & $l$ & Speed Range & Pause \\
\hline \hline Slow & $1 \mathrm{~m}$ & $1 \mathrm{~m} / \mathrm{sec}$ & $1 \mathrm{sec}$ & $1-2 \mathrm{~m} / \mathrm{sec}$ & $1 \mathrm{sec}$ \\
Moderate & $5 \mathrm{~m}$ & $5 \mathrm{~m} / \mathrm{sec}$ & $1 \mathrm{sec}$ & $5-8 \mathrm{~m} / \mathrm{sec}$ & $1 \mathrm{sec}$ \\
Fast & $15 \mathrm{~m}$ & $15 \mathrm{~m} / \mathrm{sec}$ & $1 \mathrm{sec}$ & $10-20 \mathrm{~m} / \mathrm{sec}$ & $1 \mathrm{sec}$ \\
Mixed & - & - & - & $1-20 \mathrm{~m} / \mathrm{sec}$ & $1 \mathrm{sec}$ \\
\hline
\end{tabular}

cells, each of which has a sensor node at the center. Each sensor node can detect the objects located in its grid cell and position them [31], [33]. Like in the previous work [13], [26], the radio transmission range is set at $40 \mathrm{~m}$. In order to save the energy spent in idle listening, the simulator employs the B-MAC [25] in its MAC layer. Specifically, a sensor node can send a message at any time by including a preamble. Each sensor node periodically wakes up to sample the channel. If some incoming message is detected, it stays awake to receive the message. Otherwise, the node enters the sleeping mode. The simulator also includes an energy model that measures the energy consumption of each sensor node. Approximate location queries are issued randomly from the sensor nodes in the field. The query rate is set at $1 / \mathrm{sec}$ by default. The error tolerances of the queries are randomly distributed between 0 and $p_{\max }$. The default setting of $p_{\max }$ is $50 \mathrm{~m}$.

To facilitate geographical message routing (such as for update reporting and query forwarding), the GPSR protocol [15] is employed by the simulator. GPSR operates in two modes: greedy mode and perimeter mode. In the greedy mode, a sensor node forwards a message to a neighbor closer to the destination than itself. If no such neighbor exists, the algorithm switches to the perimeter mode, which recovers by routing around the perimeter of the region.

Three mobility models, random walk, semirandom walk, and random waypoint, are used to model the moving pattern of objects in the simulation. The random walk model has been described in Section 5.1. The semirandom walk is similar to the random walk. However, unlike the random walk, after each movement step, the direction is adjusted by a random small angle $\epsilon_{\theta}$ (uniformly distributed between $\left.\left[-\frac{\pi}{8}, \frac{\pi}{8}\right]\right)$, and the speed is adjusted by a random small percentage $\epsilon_{v}$ (uniformly distributed between [ -5 percent, 5 percent]). In the random waypoint model, an object selects a destination at random in the simulated field and moves to the destination at a speed randomly chosen from a configured range; upon arrival, it pauses for a random period and selects a new destination. The random walk model is suitable for simulating small-scale scenarios, whereas the random waypoint model is more suitable for large-scale on-purpose movements [17]. The mobility profiles used in the experiments, including slow, moderate, fast, and mixed, are listed in Table 3.

We compare EASE with CS and LS (described in Section 3). The performance is measured in terms of message complexity (the number of messages transferred in the network) and energy consumption of the sensor nodes. Similar to [26], we do not measure the message overhead incurred by the underlying routing protocol (for example, beacons in GPSR). Such an overhead is usually of lower order than the application data traffic. To facilitate an easy illustration and performance comparison, we report the normalized cost for each performance metric. The normalized

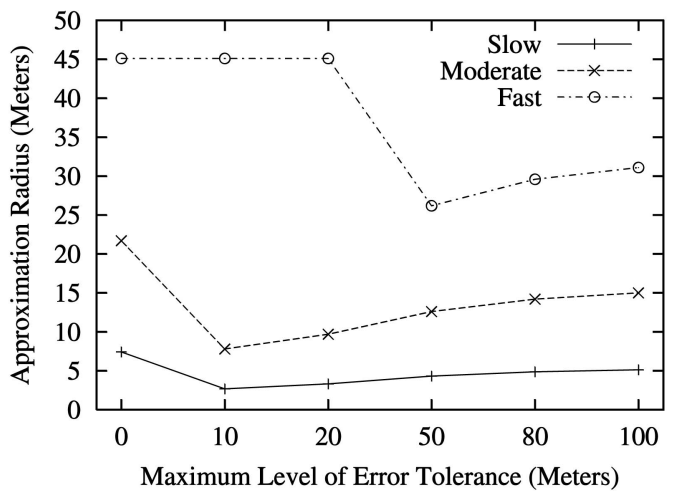

(a)



(b)

Fig. 3. Optimal setting of the approximation radius $(\lambda=1 / \mathrm{sec})$. (a) Approximation setting obtained from theoretical analysis. (b) Simulation results $\left(p_{\max }=50 \mathrm{~m}\right)$.

cost of a scheme is defined as the ratio of the measured cost of the scheme to that of CS. The smaller the normalized cost is, the better the scheme performs. Each simulation run lasted for 500 seconds of simulated time; the first 20 seconds were considered the warm-up period to eliminate initialization effects such as speed decay [34].

\subsection{Optimal Setting of the Approximation Radius}

The optimal approximation setting of EASE, $r$, can be obtained using the optimization technique described in Section 5.2 under the assumption of random walk mobility. Fig. 3a shows the optimal settings of $r$ for slow, moderate, and fast random walk mobility profiles under a number of increasing error tolerances. When the error tolerance is low (that is, $p_{\max }=0$ for slow and moderate profiles; $p_{\max } \leq 20$ for a fast profile), EASE selects a large $r\left(>p_{\max }\right)$ to suppress remote location updates. In such cases, all queries will be forwarded to the local storage nodes for high-precision answers; the selection of a large $r$ implies that the cost reduction for location updates outweighs the overhead for query forwarding. As the error tolerance becomes higher, EASE chooses a smaller $r\left(<p_{\max }\right)$ to limit query forwarding, thus reducing the overall traffic. When the error tolerance is further increased, approximation radius $r$ is slightly increased to reduce the update traffic.

Next, we compare the above approximation settings obtained from the theoretical analysis with those obtained from simulation experiments. In the simulation, we vary the approximation radius from $0,1,2, \ldots, 50$ and test the performance of each radius. The results for $p_{\max }=50$ are 


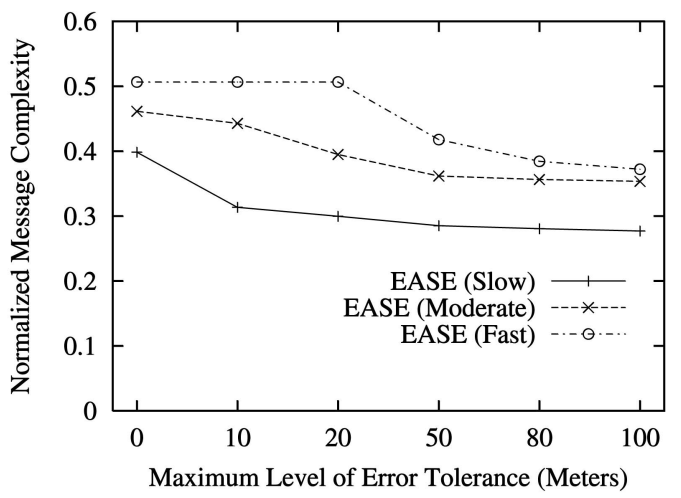

(a)

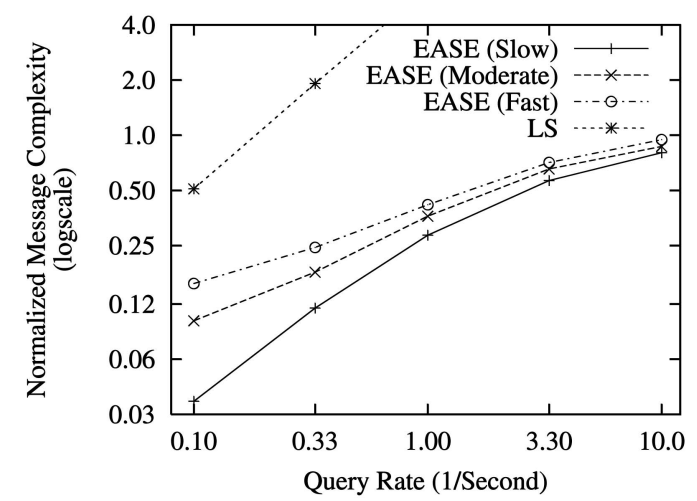

(b)

Fig. 4. Message complexity. (a) Message complexity versus query precision requirement $(\lambda=1 / \mathrm{sec})$. (b) Message complexity versus query rate $\left(p_{\max }=50 \mathrm{~m}\right)$.

plotted in Fig. 3b. The analytical approximation radii are also shown in the figure. As can be seen, the analytical approximation settings are close to the actual optimal points and approach the optimal performances. This validates our analysis of the optimal approximation setting.

\subsection{Message Complexity}

This section examines the message complexity of EASE with the optimal approximation setting. We use the random walk mobility model in this set of experiments. Fig. 4a shows the normalized message complexity under various error tolerances. Neither LS nor CS is aware of the system workload. LS has the highest message complexity, that is, 6.36, which is not plotted in the figure. In contrast, EASE is able to set a proper approximation radius in optimizing the network traffic based on the query and update patterns. As a result, EASE improves the performance over CS by 49-72 percent. Even when the error tolerance is set at zero, by selecting a large approximation radius, as shown in Fig. 3a, EASE significantly reduces the update traffic at the cost of slightly increased query traffic and achieves an improvement of 49-60 percent in the overall traffic. In general, the higher the error tolerance, the greater is the improvement achieved by EASE. This is expected since EASE is designed to exploit the error tolerance in location queries. A higher error tolerance implies more space for performance improvement. Among the three mobility profiles, the slow objects obtain the most significant performance improvement. This is mainly because of the high movement locality exhibited by the slow objects; most location

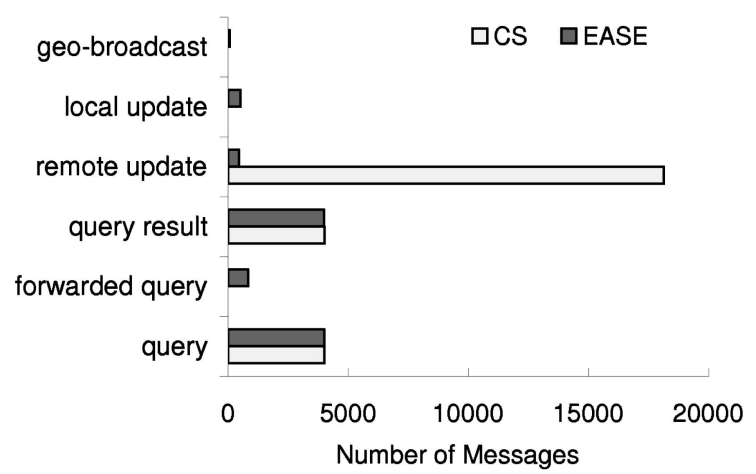

Fig. 5. Breakdown of message complexity (moderate, $p_{\max }=50 \mathrm{~m}$ ).

changes result in local updates that are propagated only to the local storage nodes.

To gain more insight into how EASE improves the performance over CS, we provide a breakdown of the traffic in Fig. 5 , where $p_{\max }$ is set at $50 \mathrm{~m}$. In the CS scheme, a significant amount of traffic contributes to remote updates. By keeping imprecise data at the centric storage node, EASE reduces remote updates by several orders of magnitude. Fig. 5 also shows that EASE's overhead for forwarding queries and geocasting is trivial compared to the reduced update traffic. Overall, EASE reduces the total message complexity of CS by 62 percent.

Fig. $4 \mathrm{~b}$ shows the normalized message complexity as a function of the query rate when $p_{\max }$ is fixed at $50 \mathrm{~m}$. LS performs the worst. For CS, when the query rate is low, most network traffic is due to location updates. EASE significantly cuts down the update traffic by setting a large approximation radius and reduces the overall message complexity by up to 96 percent. When the query rate is extremely high (for example, 10), the approximation radius is set close to zero and, hence, EASE has performance similar to CS.

\subsection{Energy Consumption}

We now proceed to evaluate the energy consumption of EASE with the optimal approximation setting. Figs. $6 a$ and $6 \mathrm{~b}$ show the total energy consumed by the sensor network during a simulation run, which is normalized by that of CS. In Fig. 6a, LS has the highest normalized energy (that is, 3.73) and is not plotted. Basically, the trends for energy consumption are similar to those for message complexity (Figs. 4a and $4 \mathrm{~b}$ ). However, the performance improvement of EASE over LS and CS is less in terms of energy consumption. This is partly because in addition to sending and receiving messages, the sensor nodes spend energy in idle listening and sleeping. The latter portion of the energy cost is similar for all schemes.

We also measure the energy consumption of each individual sensor node. The most energy-consuming node generally determines the lifetime of the sensor network, and thus, its energy consumption is used as the performance metric in Fig. 7. We can see that EASE performs the best in all cases except when the query rate is very low (leftmost points in Fig. $7 \mathrm{~b}$ ). In most cases, the energy consumption of EASE is only 20-50 percent of that of CS. This implies that EASE can prolong the network lifetime over CS by a factor of 2-5. It is also interesting to note that unlike the case of total energy consumption, EASE achieves a better performance for fast objects than moderate objects. This is 


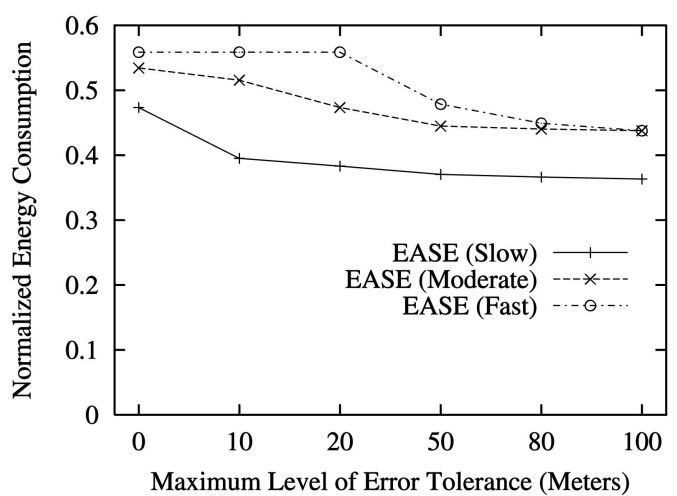

(a)

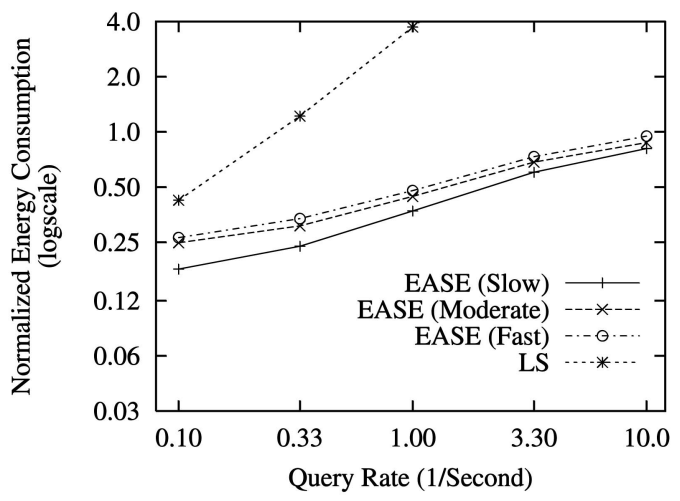

(b)

Fig. 6. Total energy consumption. (a) Energy consumption versus query precision requirement $(\lambda=1 / s e c)$. (b) Energy consumption versus query rate $\left(p_{\max }=50 \mathrm{~m}\right)$.

explained as follows: With CS, all queries and updates are sent to the centric storage nodes. Hence, the centric storage node is a hotspot. However, with reduced update traffic by EASE, the hotspot is no longer the centric storage node. Instead, some local storage nodes become hotspots. On one hand, a faster moving object generates more update traffic. On the other hand, a faster moving object changes local storage nodes more frequently and, hence, balances the loads of the sensor nodes. Combining these effects, EASE has a longer network lifetime for slow and fast objects than for moderate objects.

\subsection{Query Latency}

The CS scheme is expected to have a very good performance in query latency because the accurate location data are always available at the centric storage node; thus, a query can quickly be answered there. With the EASE scheme, if a query is not satisfied by the centric storage node due to insufficient precision, it is forwarded to the corresponding local storage node. Thus, the average querying path is lengthened due to such query forwarding. In this section, we measure the average latency of answering approximate location queries to examine how well EASE can perform in terms of query latency.

As shown in Fig. 8, when the error tolerance is low (that is, $p_{\max }=0$, the worst case), EASE performs worse than CS by no more than 52 percent. However, as the error tolerance increases, EASE consistently improves the query latency (and even outperforms CS for large values of $p_{\max }$ ). This is mainly because for a larger value of $p_{\max }$, EASE incurs less

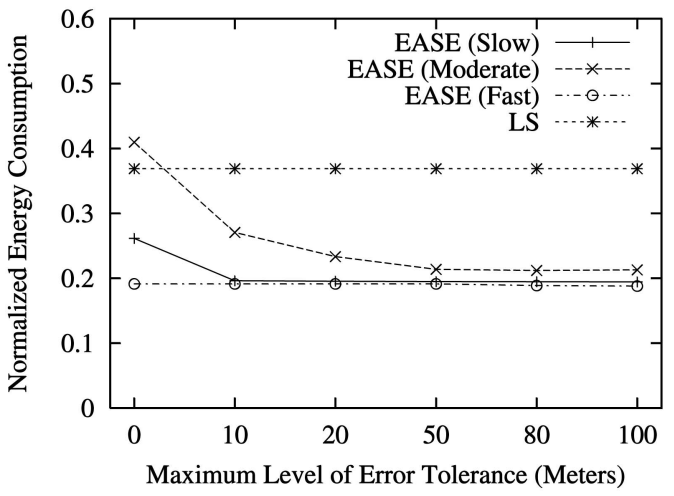

(a)

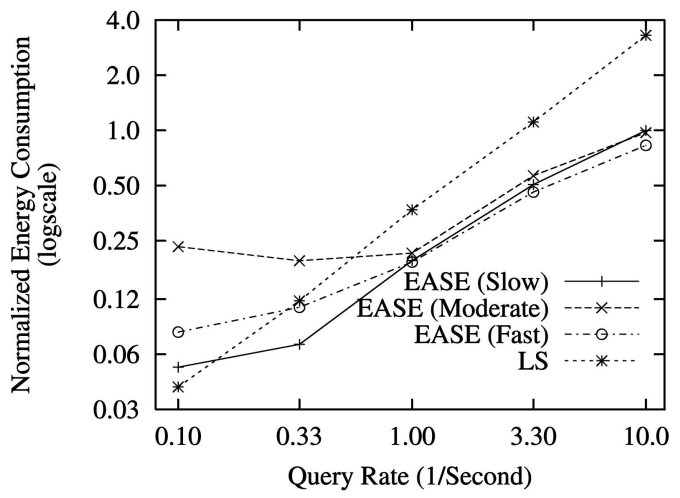

(b)

Fig. 7. Energy consumption of the most consuming node. (a) Highest energy consumption versus query precision requirement $(\lambda=1 / \mathrm{sec})$. (b) Highest energy consumption versus query rate $\left(p_{\max }=50 \mathrm{~m}\right)$.

network traffic (as shown in Fig. 4a) and, hence, less message transmission collisions, which reduces the overall latency even though the querying path is lengthened.

\subsection{Performance of the Adaptive Setting Algorithm}

Finally, we evaluate the performance of the adaptive algorithm for setting approximation radius $r$ (Section 5.3). Radius $r$ is initialized by some seed value and is updated by the proposed adaptive algorithm afterward. We set $\alpha, \epsilon$, and $\delta$ at $0.5,0.1$, and 0.1 , respectively, in the adaptive algorithm. Fig. 9 shows the normalized energy consumption for the four mobility profiles of random waypoint mobility when the maximum error tolerance $p_{\max }$ is set at $50 \mathrm{~m}$ and the query rate $\lambda$ is set at $1 /$ sec. We test two methods to seed $r$,

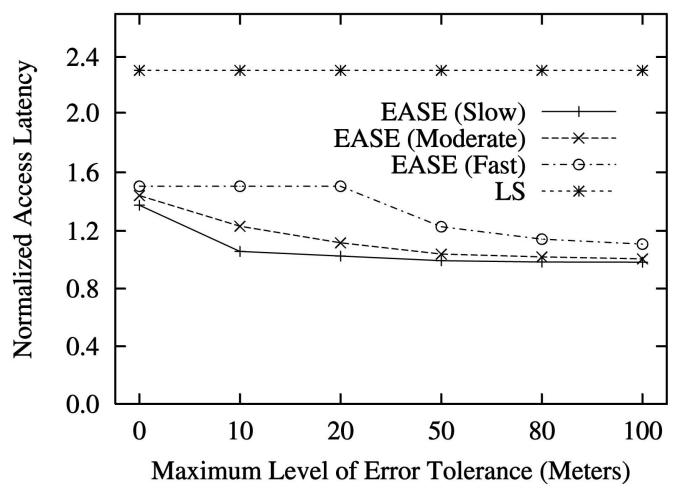

Fig. 8. Query latency versus query precision requirement $(\lambda=1 / \mathrm{sec})$. 


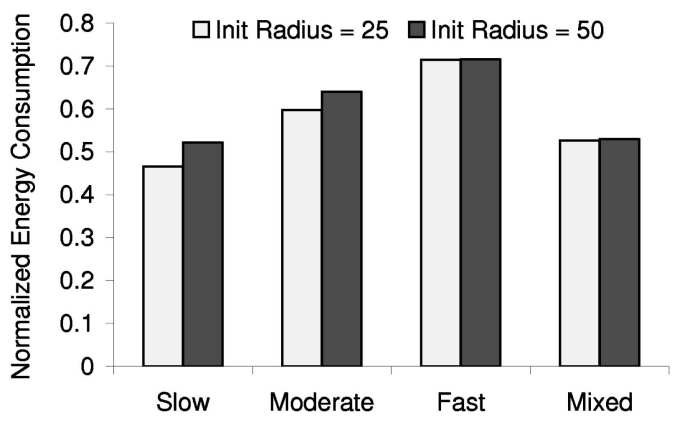

(a)

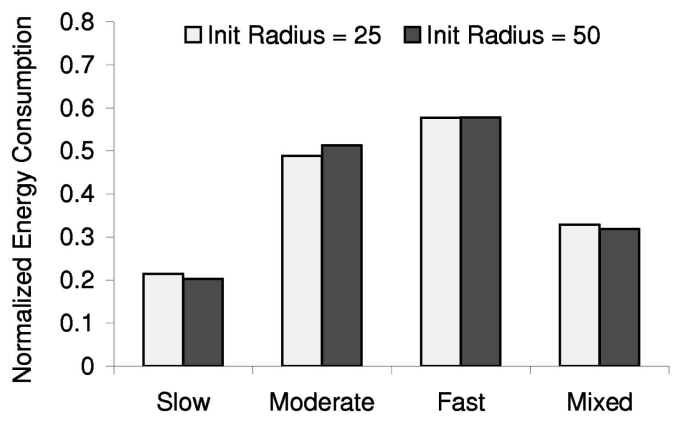

(b)

Fig. 9. Performance of adaptive settings (waypoint mobility). (a) Total energy consumption. (b) Energy consumption at the most consuming node.

that is, initializing $r$ with the average error tolerance (that is, 25 ) or the maximum error tolerance (that is, 50). As can be seen, the adaptive algorithm works effectively in both cases tested. EASE improves over CS by 30-50 percent in terms of total energy consumption, and its most consuming node spends only 20-60 percent of the energy of CS's (that is, extends the lifetime of the sensor network by a factor of 1.5$5)$. The two initial values of $r$ achieve a similar performance. Fig. 10 shows the message complexities, measured every 10 seconds, of CS and EASE (with two different initial values) as a function of simulated time. It is clear that after the warm-up period (that is, after 100 seconds), the two EASE schemes converge. This also implies that the adaptive algorithm is not sensitive to the initial setting of $r$. Similar performance trends can be observed for the semirandom mobility model. As shown in Fig. 11, EASE substantially outperforms CS in terms of energy consumption.

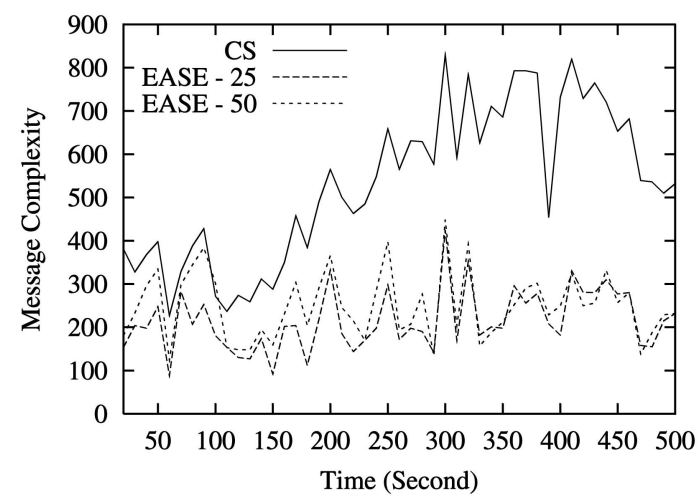

Fig. 10. Message complexity along time series (waypoint, slow mobility profile).

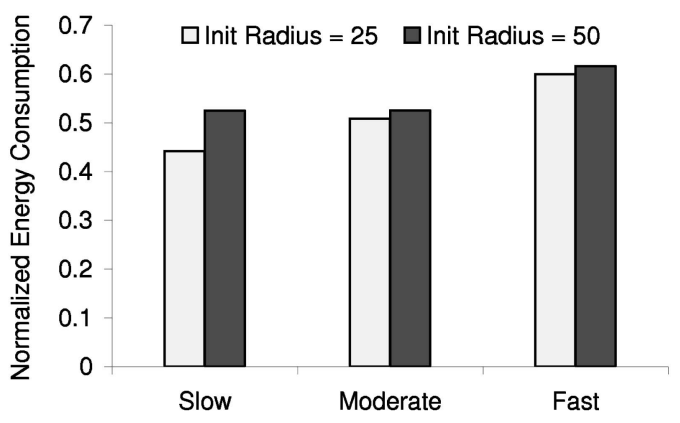

(a)

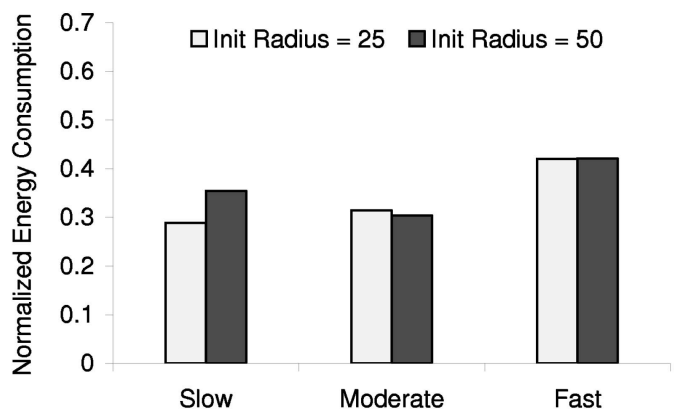

(b)

Fig. 11. Performance of adaptive settings (semirandom mobility). (a) Total energy consumption. (b) Energy consumption at the most consuming node.

\section{Conclusions}

This paper presents a study on the processing of precisionconstrained approximate location queries for object-tracking sensor networks. An energy-efficient storage scheme called EASE has been developed to efficiently reduce the network traffic, conserve the energy of sensor nodes, and improve the network lifetime. We have analyzed the optimal setting of the approximation radius when the object mobility pattern is known and devised an adaptive algorithm to adjust the approximation setting when the mobility pattern is not available or is dynamically changing. We have also evaluated the proposed techniques through extensive simulation-based experiments. The experimental results validated our theoretical analysis of the optimal approximation setting. They also demonstrated that the EASE scheme, working together with the proposed approximation setting methods, saves significant energy for sensor networks and prolongs the network lifetime.

With regard to future work, we plan to extend EASE to answering approximate spatial queries such as finding $k$ nearest neighbors. Besides one-shot queries, we will also investigate continuous monitoring queries. In addition, we are planning to develop a testbed using Berkeley Motes to validate the proposed schemes.

\section{APPENDIX}

\section{ANALYSIS OF THE TWO-DIMENSIONAL RANDOM WaLK MODEL}

Consider a two-dimensional random walk model in which at each step, an object moves a distance of $d$ in a direction uniformly distributed in $[0,2 \pi)$. Assume that the object starts a random walk from point $O$. We divide the plane into a set of 




Fig. 12. A set of rings.

rings of sufficiently small width $\Delta$. As shown in Fig. 12, ring 0 is enclosed by a circle centered at $O$ with radius $\frac{1}{2} \Delta$, that is, ring 0 contains all points that are within distance $\frac{1}{2} \Delta$ from $O$. For each $i \geq 1$, ring $i$ is enclosed by two circles centered at $O$ with radii $\left(i-\frac{1}{2}\right) \Delta$ and $\left(i+\frac{1}{2}\right) \Delta$, respectively, that is, ring $i$ includes all points that are $\left(i-\frac{1}{2}\right) \Delta$ to $\left(i+\frac{1}{2}\right) \Delta$ away from $O$.

Without loss of generality, we assume that $d=k \Delta$, where $k$ is an integer. Consider an object located in ring $i$. We approximate its distance to $O$ as $\Phi=i \Delta$. Fig. 13 shows that if the object moves in direction $\theta$, its new distance to $O$ is given by

$$
\begin{aligned}
\Phi^{\prime} & =\sqrt{(d \sin \theta)^{2}+(i \Delta+d \cos \theta)^{2}} \\
& =\sqrt{d^{2}+(i \Delta)^{2}+2 d i \Delta \cos \theta} \\
& =\Delta \cdot \sqrt{k^{2}+i^{2}+2 k i \cos \theta} .
\end{aligned}
$$

The object is located in ring $j$ after the move if and only if

$$
\left(j-\frac{1}{2}\right) \Delta \leq \Delta \cdot \sqrt{k^{2}+i^{2}+2 k i \cos \theta} \leq\left(j+\frac{1}{2}\right) \Delta,
$$

or, equivalently,

$$
\frac{\left(j-\frac{1}{2}\right)^{2}-i^{2}-k^{2}}{2 k i} \leq \cos \theta \leq \frac{\left(j+\frac{1}{2}\right)^{2}-i^{2}-k^{2}}{2 k i} .
$$

If $j<|i-k|$ or $j>i+k$, the above condition is impossible to satisfy. In this case, the transition probability from ring $i$ to $j$ is given by 0 .

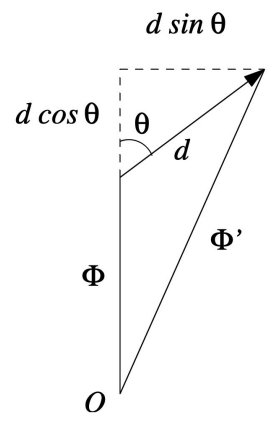

Fig. 13. Distance computation.

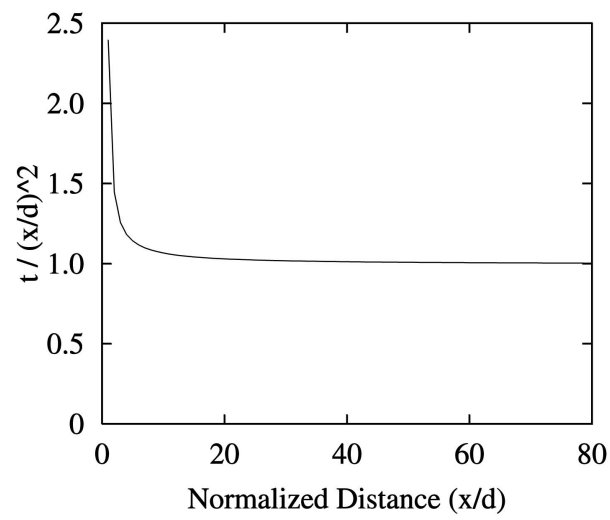

Fig. 14. Simulation results of a random walk.

If $j=|i-k|$, the new distance can be in the range $\left[j \Delta,\left(j+\frac{1}{2}\right) \Delta\right]$ only. Due to symmetry, only the range $[0, \pi]$ needs to be considered for $\theta$. Thus, the transition probability from ring $i$ to $j$ is given by

$$
\frac{1}{\pi} \cdot\left(\arccos \frac{j^{2}-i^{2}-k^{2}}{2 k i}-\arccos \frac{\left(j+\frac{1}{2}\right)^{2}-i^{2}-k^{2}}{2 k i}\right) .
$$

If $j=i+k$, the new distance can be in the range [ $(j-$ $\left.\left.\frac{1}{2}\right) \Delta, j \Delta\right]$ only. Hence, the transition probability from ring $i$ to $j$ is given by

$$
\frac{1}{\pi} \cdot\left(\arccos \frac{\left(j-\frac{1}{2}\right)^{2}-i^{2}-k^{2}}{2 k i}-\arccos \frac{j^{2}-i^{2}-k^{2}}{2 k i}\right) .
$$

If $|i-k|<j<i+k$, the new distance can be in the range $\left[\left(j-\frac{1}{2}\right) \Delta,\left(j+\frac{1}{2}\right) \Delta\right]$. Therefore, the transition probability from ring $i$ to $j$ is given by

$$
\frac{1}{\pi} \cdot\left(\arccos \frac{\left(j-\frac{1}{2}\right)^{2}-i^{2}-k^{2}}{2 k i}-\arccos \frac{\left(j+\frac{1}{2}\right)^{2}-i^{2}-k^{2}}{2 k i}\right) .
$$

We model each ring as a state and represent the object location during a random walk as a probability vector $\left[p_{0}, p_{1}, p_{2}, \ldots\right]$, where $p_{i}$ is the probability that the object is located in ring $i$. Starting from the vector $[1,0,0, \ldots]$ (that is, the object starts a random walk from point $O$ ), the vector after each move can be computed iteratively with the above transition probabilities. It is then easy to calculate the probability of the object's first move beyond a given ring $i$ (that is, distance $x=\left(i+\frac{1}{2}\right) \Delta$ from $O$ ) at each step. In this way, the average number of steps $t$ the object takes to first move beyond $x$ away from $O$ can be derived numerically.

Fig. 14 shows the simulation results, where the $x$-axis represents the normalized distance $x / d$ from the starting point $O$, and the $y$-axis represents the ratio of $t$ to $(x / d)^{2}$. As seen in Fig. 14, when $x$ is beyond a few times $d$, the ratio approaches a constant 1 . Therefore, $t$ can be approximated by $(x / d)^{2}$.

\section{ACKNOWLEDGMENTS}

This work was supported by the Research Grants Council of Hong Kong under Projects HKBU211505, HKBU211206, HKBU211307, and FRG/05-06/II-65. Xueyan Tang's work 
was supported in part by Nanyang Technological University under Project RG47/06. Wang-Chien Lee was supported in part by the US National Science Foundation under Grants IIS-0328881, IIS-0534343, and CNS-0626709. A preliminary report of this work appeared in the Proceedings of the Second IEEE Conference on Sensor and Ad Hoc Communications and Networks (SECON) [37].

\section{REFERENCES}

[1] The Network Simulator-ns-2, http://www.isi.edu/nsnam/ns/, 2007.

[2] NRL's Sensor Network Extension to ns-2, http://nrlsensorsim. pf.itd.nrl.navy.mil/, 2007.

[3] K. Akkaya and M. Younis, "A Survey of Routing Protocols in Wireless Sensor Networks," Ad Hoc Networks, 2004.

[4] I.F. Akyildiz, J. McNair, J. Ho, H. Uzunalioglu, and W. Wang, "Mobility Management for Next Generation Wireless Systems," Proc. IEEE, vol. 87, no. 8, pp. 1347-1384, Aug. 1999.

[5] A. Deligiannakis, Y. Kotidis, and N. Roussopoulos, "Compressing Historical Information in Sensor Networks," Proc. ACM SIGMOD '04, pp. 527-538, 2004.

[6] A. Deligiannakis, Y. Kotidis, and N. Roussopoulos, "Processing Approximate Aggregate Queries in Wireless Sensor Networks," Information Systems, vol. 31, no. 8, pp. 770-792, Dec. 2006.

[7] A. Demers, J. Gehrke, R. Rajaraman, J. Trigoni, and Y. Yao, "The Cougar Project: A Work-in-Progress Report," ACM SIGMOD Record, vol. 32, no. 4, Dec. 2003.

[8] A. Ghose, J. Grossklags, and J. Chuang, "Resilient Data-Centric Storage in Wireless Ad-Hoc Sensor Networks," Proc. Fourth Int'l Conf. Mobile Data Management (MDM '03), Jan. 2003.

[9] S. Goel and T. Imielinski, "Prediction-Based Monitoring in Sensor Networks: Taking Lessons from MPEG," ACM Computer Comm. Rev., vol. 31, no. 5, Oct. 2001.

[10] C. Gui and P. Mohapatra, "Power Conservation and Quality of Surveillance in Target Tracking Sensor Networks," Proc. ACM MobiCom '04, Oct. 2004.

[11] Q. Han, S. Mehrotra, and N. Venkatasubramanian, "Energy Efficient Data Collection in Distributed Sensor Environments," Proc. 24th Int'l Conf. Distributed Computing Systems (ICDCS '04), Mar. 2004.

[12] W. Heinzelman, A. Chandrakasan, and H. Balakrishnan, "EnergyEfficient Communication Protocol for Wireless Sensor Networks," Proc. 33rd Hawaii Int'l Conf. System Sciences (HICSS '00), Jan. 2000.

[13] C. Intanagonwiwat, R. Govindan, and D. Estrin, "Directed Diffusion: A Scalable and Robust Communication Paradigm for Sensor Networks," Proc. ACM MobiCom '00, Aug. 2000.

[14] H. Jiang and S. Jin, "Scalable and Robust Aggregation Techniques for Extracting Statistical Information in Sensor Networks," Proc. 26th Int'l Conf. Distributed Computing Systems (ICDCS '06), July 2006.

[15] B. Karp and H.T. Kung, "GPSR: Greedy Perimeter Stateless Routing for Wireless Sensor Networks," Proc. ACM MobiCom '00, Aug. 2000.

[16] S. Kim, R. Fonseca, and D. Culler, "Reliable Transfer on Wireless Sensor Networks," Proc. First IEEE Conf. Sensor and Ad Hoc Comm. and Networks (SECON '04), Oct. 2004.

[17] H.T. Kung and D. Vlah, "Efficient Location Tracking Using Sensor Networks," Proc. IEEE Wireless Comm. and Networking Conf. (WCNC'03), Mar. 2003.

[18] H. Liu, X. Jia, P. Wan, C.-W. Yi, S. Makki, and P. Niki, "Maximizing Lifetime of Sensor Surveillance Systems," IEEE/ ACM Trans. Networking, 2006.

[19] C. Lu, G. Xing, O. Chipara, C.-L. Fok, and S. Bhattacharya, "A Spatiotemporal Query Service for Mobile Users in Sensor Networks," Proc. 25th Int'l Conf. Distributed Computing Systems (ICDCS '05), June 2005.

[20] S.R. Madden, M.J. Franklin, J.M. Hellerstein, and W. Hong, "TinyDB: An Acquisitional Query Processing System for Sensor Networks," ACM Trans. Database Systems, vol. 30, no. 1, pp. 122173, 2005.

[21] S. Nath, P.B. Gobbons, S. Seshan, and Z.R. Anderson, "Synopsis Diffusion for Robust Aggregation in Sensor Networks," Proc. Second ACM Int'l Conf. Embedded Networked Sensor Systems (SenSys '04), Nov. 2004.
[22] J.C. Navas and T. Imielinski, "GeoCast-Geographic Addressing and Routing," Proc. ACM MobiCom, 1997.

[23] C. Olston, B.T. Loo, and J. Widom, "Adaptive Precision Setting for Cached Approximate Values," Proc. ACM SIGMOD '01, May 2001.

[24] S. Pattem, S. Poduri, and B. Krishnamachari, "Energy-Quality Tradeoffs for Target Tracking in Wireless Sensor Networks," Proc. Second Int'l Workshop Information Processing in Sensor Networks (IPSN '03), Apr. 2003.

[25] J. Polastre, J. Hill, and D. Culler, "Versatile Low Power Media Access for Wireless Sensor Networks," Proc. ACM Second Int'l Conf. Mobile Systems, Applications, and Services (MobiSys '04), Nov. 2004.

[26] S. Ratnasamy, B. Karp, S. Shenker, D. Estrin, R. Govindan, L. Yin, and F. Yu, "Data-Centric Storage in Sensornets with GHT, a Geographic Hash Table," ACM/Kluwer Mobile Networks and Applications, vol. 8, no. 4, 2003.

[27] M.A. Sharaf, J. Beaver, A. Labrinidis, and P.K. Chrysanthis, "Balancing Energy Efficiency and Quality of Aggregate Data in Sensor Networks," VLDB J., vol. 13, no. 4, Dec. 2004.

[28] N. Shrivastava, C. Buragohain, and D. Agrawal, "Medians and Beyond: New Aggregation Techniques for Sensor Networks," Proc. Second ACM Int'l Conf. Embedded Networked Sensor Systems (SenSys '04), Nov. 2004.

[29] D. Smith and S. Singh, "Approaches to Multisensor Data Fusion in Target Tracking: A Survey," IEEE Trans. Knowledge and Data Eng., vol. 18 , no. 12, pp. 1696-1710, Dec. 2006.

[30] X. Tang and J. Xu, "Extending Network Lifetime for PrecisionConstrained Data Aggregation in Wireless Sensor Networks," Proc. IEEE INFOCOM '06, Apr. 2006.

[31] Q.X. Wang, W.P. Chen, R. Zheng, K. Lee, and L. Sha, "Acoustic Target Tracking Using Tiny Wireless Sensor Devices," Proc. Second Int'l Workshop Information Processing in Sensor Networks (IPSN '03), Apr. 2003.

[32] Y. Xu, J. Winter, and W.-C. Lee, "Dual Prediction-Based Reporting Mechanism for Object Tracking Sensor Networks," Proc. First Ann. Int'l Conf. Mobile and Ubiquitous Systems (MobiQuitous '04), Aug. 2004.

[33] H. Yang and B. Sikdar, "A Protocol for Tracking Mobile Targets Using Sensor Networks," Proc. IEEE Workshop Sensor Network Protocols and Applications, May 2003.

[34] J. Yoon, M. Liu, and B. Noble, "Sound Mobility Models," Proc. ACM MobiCom '03, Sept. 2003.

[35] W. Zhang and G. Cao, "Optimizing Tree Reconfiguration for Mobile Target Tracking in Sensor Networks," Proc. IEEE INFOCOM '04, Mar. 2004.

[36] W. Zhang, G. Cao, and T.L. Porta, "Data Dissemination with RingBased Index for Wireless Sensor Networks," Proc. 11th IEEE Int'l Conf. Network Protocols (ICNP '03), Nov. 2003.

[37] J. Xu, X. Tang, and W-C. Lee, "EASE: An Energy-Efficient InNetwork Storage Scheme for Object Tracking in Sensor Networks," Proc. Second IEEE Conf. Sensor and Ad Hoc Comm. and Networks (SECON '05), Sept. 2005.

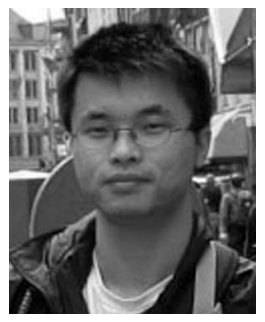

Jianliang $\mathrm{Xu}$ received the BEng degree in computer science and engineering from Zhejiang University, Hangzhou, China, in 1998 and the $\mathrm{PhD}$ degree in computer science from the Hong Kong University of Science and Technology in 2002. He is currently an assistant professor in the Department of Computer Science, Hong Kong Baptist University. His research interests include mobile and pervasive computing, wireless sensor networks, and distributed systems, with an emphasis on data management. He has published more than 50 technical papers in these areas, most of which appeared in prestigious journals and conference proceedings, including ACM SIGMOD, the ACM International Conference on Mobile Systems, Applications, and Services (MobiSys), the IEEE International Conference on Data Engineering (ICDE), IEEE INFOCOM, IEEE Transactions on Parallel and Distributed Systems, and IEEE Transactions on Knowledge and Data Engineering. He is an editor of a book entitled Web Content Delivery, published by Springer. He has also served as a program committee member for many international conferences. He is a member of the IEEE. 


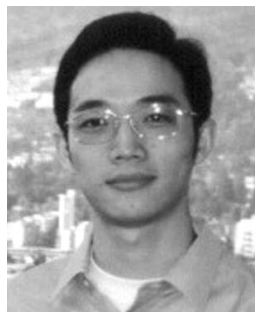

Xueyan Tang received the BEng degree in computer science and engineering from Shanghai Jiao Tong University, Shanghai, China, in 1998 and the PhD degree in computer science from the Hong Kong University of Science and Technology in 2003. He is currently an assistant professor in the School of Computer Engineering, Nanyang Technological University, Singapore. His research interests include mobile and pervasive computing, wireless sensor networks, Web and Internet, and distributed systems, particularly the data management aspects in these areas. He has published more than 30 papers in prestigious journals and conference proceedings. $\mathrm{He}$ is an editor of a book entitled Web Content Delivery, published by Springer. $\mathrm{He}$ has also served as a program committee member for many international conferences. $\mathrm{He}$ is a member of the IEEE.

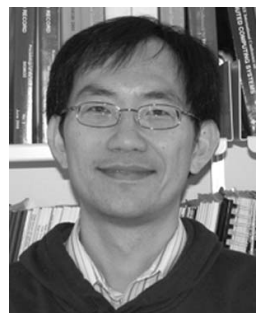

Wang-Chien Lee received the BS degree from the Information Science Department, National Chiao Tung University, Taiwan, the MS degree from the Computer Science Department, Indiana University, and the PhD degree from the Computer and Information Science Department, Ohio State University. $\mathrm{He}$ is an associate professor of computer science and engineering at Pennsylvania State University. Prior to joining Pennsylvania State University, he was a principal member of the technical staff at Verizon/GTE Laboratories, Inc. He leads the Pervasive Data Access (PDA) Research Group at Pennsylvania State University to perform cross-area research in database systems, pervasive/mobile computing, and networking. He is particularly interested in developing data management techniques (including accessing, indexing, caching, aggregation, dissemination, and query processing) for supporting complex queries in a wide spectrum of networking and mobile environments such as peer-to-peer networks, mobile ad hoc networks, wireless sensor networks, and wireless broadcast systems. Meanwhile, he has worked on XML, security, information integration/retrieval, and object-oriented databases. His research has been supported by the US National Science Foundation and industry grants. Most of his research result has been published in prestigious journals and conference proceedings in the fields of databases, mobile computing, and networking. He has served as a guest editor for several journal special issues on mobile databaserelated topics, including IEEE Transactions on Computers, IEEE Personal Communications Magazine, ACM Mobile Networks and Applications, and ACM Wireless Networks. He was the founding program committee cochair for the International Conference on Mobile Data Management. He is a member of the IEEE and the ACM.

$\triangleright$ For more information on this or any other computing topic, please visit our Digital Library at www.computer.org/publications/dlib. 